\title{
Synaptic Strengthening Mediated by Bone Morphogenetic Protein-Dependent Retrograde Signaling in the Drosophila CNS
}

\author{
Richard A. Baines \\ Neuroscience Group, Department of Biological Sciences, University of Warwick, Coventry, CV4 7AL, United Kingdom
}

\begin{abstract}
Retrograde signaling is an essential component of synaptic development and physiology. Previous studies show that bone morphogenetic protein (BMP)-dependent retrograde signaling is required for the proper development of the neuromuscular junction (NMJ) in Drosophila. These studies, moreover, raised the significant possibility that the development of central motor circuitry might similarly be reliant on such signaling. To test this hypothesis, retrograde signaling between postsynaptic motoneurons and their presynaptic interneurons is examined. Postsynaptic expression of an adenylate cyclase encoded by rutabaga ( rut $)$, is sufficient to strengthen synaptic transmission at these identified central synapses. Results are presented to show that the underlying mechanism is dependent on BMP retrograde signaling. Thus, presynaptic expression of an activated TGF- $\beta$ receptor, thickvien $(t k v)$, or postsynaptic expression of a TGF- $\beta$ ligand, glass-bottom boat ( $g b b$ ), is sufficient to phenocopy strengthening of synaptic transmission. In the absence of $g b b$, endogenous synaptic transmission is significantly weakened and, moreover, postsynaptic overexpression of rut is unable to potentiate synaptic function. Potentiation of presynaptic neurotransmitter release, mediated by increased postsynaptic expression of $g b b$, is dependent on normal cholinergic activity, indicative that either the secretion of this retrograde signal, or its transduction, is activity dependent. Thus, in addition to the development of the NMJ and expression of myoactive FMRFamide-like peptides in specific central neurons, the results of the present study indicate that this retrograde signaling cascade also integrates the development and function of central motor circuitry that controls movement in Drosophila larvae.
\end{abstract}

Key words: aCC; Gbb; neural activity; retrograde signaling; RP2; synaptic development

\section{Introduction}

The establishment, growth, and plasticity of synaptic connectivity are critically dependent on the transfer of signaling molecules between presynaptic and postsynaptic cells. This signaling can be both anterograde or retrograde in direction. Examples of anterograde signaling include the release of Wingless $(\mathrm{Wg})$ protein by motoneurons at the Drosophila neuromuscular junction (NMJ). Blockade of Wg secretion results in reduction of synaptic growth during larval development, a time when the NMJ undergoes significant expansion (Packard et al., 2002, 2003). Retrograde signals, which influence presynaptic differentiation, maturation, and neurotransmitter release properties, include secreted molecules (i.e., the neurotrophins and Wnt proteins), diffusible gases (i.e., nitric oxide), and membrane lipids (i.e., arachidonic acid) (Fitzsimonds and Poo, 1998; Sheng and Kim, 2002; Packard et al., 2003).

Received March 29, 2004; revised June 23, 2004; accepted June 23, 2004.

This study was funded by grants from the Wellcome Trust and Royal Society. I am grateful to J. Jaynes and M. Fujioka for providing RN2 GAL4 flies. I thank E. Bier, G. Davies, M. O'Connor, P. Salvaterra, S. Selleck, S. Sweeney, K. Wharton, T. Zars, and the Bloomington Stock Center for providing flies. I thank C. Mee for quantification of $g b b$ mRNA. I acknowledge K. Moffat and M. Wall for comments.

Correspondence should be addressed to Dr. R. A. Baines, Department of Biological Sciences, University of Warwick, Coventry, CV4 7AL, UK. E-mail: RBaines@bio.warwick.ac.uk.

DOI:10.1523/JNEUROSCI.1978-04.2004

Copyright $\odot 2004$ Society for Neuroscience $\quad 0270-6474 / 04 / 246904-08 \$ 15.00 / 0$
Mutagenic screens in Drosophila identified Wishful thinking (Wit), a type II bone morphogenetic protein (BMP) receptor, to be required for development of the NMJ (Aberle et al., 2002; Marqués et al., 2002). Presynaptic, but not postsynaptic, rescue of wit nulls, with a full-length wit transgene is sufficient to rescue the defects observed in synaptic development and function, indicative that BMP proteins fulfill retrograde signaling functions at this peripheral synapse. The most likely candidate BMP signal is glass-bottom boat (Gbb), null mutations of which phenocopy the reduced synaptic structure and transmission observed in alleles of wit (Marqués et al., 2003; McCabe et al., 2003). Gbb is a ligand of the TGF- $\beta$ signaling system that is conserved across species (Massague, 1998; Massague and Wotton, 2000). Briefly, secreted ligand dimerizes and is able to bind a type II receptor (including wit in Drosophila) that then recruits a type I receptor (including tkv and sax in Drosophila) to the complex. Recruitment initiates phosphorylation of the type I receptor that in turn phosphorylates a receptor-Smad (R-Smad), which is then empowered to translocate into the nucleus to affect gene transcription.

BMPs and their receptors are expressed in the vertebrate CNS (Soderstrom et al., 1996) and, indeed, in situ analysis shows expression of wit in both the embryonic and larval CNS of Drosophila (Aberle et al., 2002; Marqués et al., 2002). Such localization is consistent with BMP signaling being involved in the development of central synapses, in addition to the peripheral NMJ. To 
test this hypothesis, I have examined the role of BMP signaling at an identified motoneuron synapse in the CNS of Drosophila.

I show that overexpression of an adenylate cyclase (rutabaga) in postsynaptic motoneurons (termed aCC and RP2) is sufficient to increase synaptic transmission, whereas increased presynaptic expression is not. Potentiation of synaptic transmission can also be induced by presynaptic expression of the activated BMP type I receptor $t k v$, or by postsynaptic expression of $g b b$. That postsynaptic activity of an adenylate cyclase is upstream of secreted $g b b$ is demonstrated by the finding that overexpression of UAS-rut in motoneurons fails to potentiate synaptic transmission in the absence of $g b b$. Taken together, the results are consistent with an activity-dependent retrograde signaling mechanism, using $g b b$ and its cognate receptors, which potentiates synaptic transmission within the CNS.

\section{Materials and Methods}

Fly stocks. Flies were fed on apple juice agar supplemented with yeast. Wild-type was Canton-S. RN2-O and RN2-E GAL4 (homozygous viable, second and third chromosome, respectively) were used to selectively express UAS-driven transgenes in aCC and RP2 (Fujioka et al., 1999; Baines, 2003). Expression of RN2 GAL4 begins in early stage 16 embryos, a stage that precedes the onset of synaptogenesis (Baines and Bate, 1998). Levels of cAMP were increased in specific neurons by expression of UAS-rut (a type I, $\mathrm{Ca}^{2+}$-calmodulin-dependent, adenylate cyclase) (Zars et al., 2000). Protein kinase A (PKA) activity was promoted using UAS-PKAact ${ }^{l}$ and inhibited using UAS-PKAinh ${ }^{1}$ (formerly termed PKA $^{\text {BDK22 }}$ ), these transgenes are described in the study by Davis et al. (1998). The $g b b^{1}$ and $g b b^{2}$ stocks are described in Wharton et al. (1999). These stocks were rebalanced over Cyo::GFP to allow unequivocal identification in early larvae. UAS- $g b b$ was obtained from E. Bier (University of California, San Diego, La Jolla, CA), whereas UAS- $d p p$ was obtained from Bloomington (stock number 1486). Constitutively active UAS-tkv (1B3) and UAS$\operatorname{saxA}$ were provided by M. O'Connor (University of Minnnesota, Minneapolis, MN). B19-Gal4 contains the entire ( $7.4 \mathrm{~kb}$ ) promoter sequence for choline acetyltransferase, the gene encoding the synthetic enzyme for $\mathrm{ACh}$ and expresses selectively in cholinergic neurons (Salvaterra and Kitamoto, 2001). Cholinergic signaling was manipulated using a temperature-sensitive allele of choline acetyltransferase (cha). Activity of this enzyme in $c h a^{t s 2}$ is reduced relative to wild-type (WT) at permissive temperatures of $\leq 20^{\circ} \mathrm{C}$, and is entirely blocked at the restrictive temperature of $29^{\circ} \mathrm{C}$ (Salvaterra and McCaman, 1985; Baines et al., 2001).

Embryo dissection. Larvae were dissected, and central neurons were accessed as described in Baines and Bate (1998). The larva was viewed using a water immersion lens (total magnification, $800 \times$ ) combined with Nomarski optics (BX51 WI microscope; Olympus Optical, Tokyo, Japan).

Electrophysiology. All recordings were performed in young first instar larvae, $1-4 \mathrm{hr}$ after hatching, at room temperature $\left(22-24^{\circ} \mathrm{C}\right)$, unless stated otherwise. Whole-cell recordings (current and voltage clamp) were achieved using thick-walled borosilicate glass electrodes (GC100TF-10; Harvard Apparatus, Edenbridge, UK), fire-polished to resistances of between 15 and $20 \mathrm{M} \Omega$. Recordings were made using an Axopatch-1D amplifier controlled by pClamp 8.1 via a Digidata 1322A (Axon Instruments, Foster City, CA). Only cells with an input resistance $>1 \mathrm{G} \Omega$ were accepted for analysis. Cells were initially identified based on both size and dorsal position in the ventral nerve cord. Unequivocal identification was determined by labeling with sulfur rhodamine $(0.3 \%$; Molecular Probes, Eugene, OR), which was included in the patch saline. Traces were filtered at $2 \mathrm{kHz}$ and sampled at $20 \mathrm{kHz}$. Spontaneous miniature currents were recorded in the presence of TTX $(0.1 \mu \mathrm{M}$; Alomone Labs, Jerusalem, Israel). Miniature currents were discriminated based on a fast rise and slower decay and on having an amplitude of at least twice background (2-3 pA). After breakthrough, currents were measured for a period of time ranging from between at least $2 \mathrm{~min}$ and for no longer than 5 min from a minimum of six cells for each genotype tested. Although currents can be recorded for longer than 5 min, cells often begin to show increased leak currents after this duration of whole-cell recording. Amplitudes of both evoked and miniature currents were measured using Minianalysis 5.2.8 (Synaptosoft, Decatur, GA).

Solutions. External saline consisted of (in $\mathrm{mm}$ ): $\mathrm{NaCl}$ (135), $\mathrm{KCl}$ (5), $\mathrm{MgCl}_{2} \cdot 6 \mathrm{H}_{2} \mathrm{O}$ (4), $\mathrm{CaCl}_{2} \cdot 2 \mathrm{H}_{2} \mathrm{O}$ (2), $\mathrm{N}$-Tris[hydroxymethyl]methyl-2amonoethanesulfonic acid (TES; 5), and sucrose (36), pH 7.15. Internal patch solution consisted of (in $\mathrm{mM}): \mathrm{K}^{+}$methylsulfonate $\left(\mathrm{KCH}_{3} \mathrm{SO}_{3}\right.$, 140), $\mathrm{MgCl}_{2} \cdot 6 \mathrm{H}_{2} \mathrm{O}$ (2), EGTA (2), $\mathrm{KCl}$ (5), and HEPES (20), pH 7.4.

Statistics. Data were compared using a nonpaired $t$ test. Results were deemed significant at $p \leq 0.05\left(^{*}\right), p \leq 0.01\left(^{* *}\right)$, or $p \leq 0.001\left(^{* * *}\right)$. All values shown are mean \pm SE. Where applicable, both the number of individual currents and number of cells recorded from are provided.

\section{Results}

Voltage-clamp recordings $\left(V_{\mathrm{h}},-60 \mathrm{mV}\right)$, from either aCC or RP2 (no differences observed between neurons) reveal the presence of large (50-300 pA) inward synaptic currents that are relatively long-lived (500-1000 msec) (Fig. 1A). These excitatory currents, which are cholinergic in nature, arise from the synchronous activity of interneurons presynaptic to these motoneurons (Baines et al., 1999; Baines, 2003). In a previous study, it was shown that the amplitude of these synaptic currents, but not their frequency, is significantly increased in alleles of dunce $(d n c)$ (Baines, 2003). Because $d n c$ affects all neurons, it was not possible in this previous study to localize the site of action to either the presynaptic interneurons or postsynaptic motoneurons.

\section{Postsynaptic cAMP-PKA is sufficient to enhance synaptic excitation of motoneurons}

To address the site of action of $d n c$ to increase synaptic transmission at this identified synapse, I used the GAL4-UAS system to target expression of rut (a type $\mathrm{I}, \mathrm{Ca}^{2+}$-calmodulin-dependent, adenylate cyclase) (Zars et al., 2000) to either the presynaptic interneurons or the postsynaptic motoneurons. Postsynaptic expression of rut, in aCC/RP2 (RN2-GAL4), is sufficient to phenocopy the effects observed in $d n c$ mutants (Baines, 2003). Thus, after postsynaptic expression of rut, the averaged amplitude of synaptic currents recorded in aCC/RP2 increased significantly compared with control $(81.9 \pm 4.2 \mathrm{pA}, 385$ currents per 8 cells vs $109 \pm 2.8$ pA, 441 currents per 8 cells; $p \leq 0.001$; RN2 vs RN2 $\times$ UAS-rut) (Fig. 1Aii,B). Overall, frequency of individual synaptic currents was not significantly different $(23 \pm 2.7$ vs $23 \pm 2.2$ per minute, respectively). Cumulative probability plots of individual synaptic currents, which better show the range of current amplitudes, reveal that there is a significant increase in the majority of individual current amplitudes compared with control (Fig. $1 \mathrm{Bii}$ ). In contrast, expression of UAS-rut in presynaptic cholinergic interneurons (B19-GAL4), which includes all those interneurons known to synaptically excite aCC/RP2 (Baines et al., 1999), did not effect synaptic current amplitude recorded in aCC/RP2 $(72.4 \pm 3.1 \mathrm{pA}, 272 / 8$ vs $76.1 \pm 2.6 \mathrm{pA}, 274 / 8 ; p>0.05 ; \mathrm{B} 19$ vs B19 $\times$ UAS-rut) $($ Fig. 1C). Thus, I conclude that the increase in synaptic current amplitude observed in alleles of $d n c$ (Baines, 2003) can be mimicked by overexpression of an adenylate cyclase in postsynaptic motoneurons.

There are a number of plausible mechanisms that would potentially evoke an increase in synaptic transmission between presynaptic interneurons and aCC/RP2. These include an increase in evoked release of ACh from presynaptic terminals, an increased postsynaptic sensitivity to ACh, an increase in number of synaptic connections between these two sets of neurons and, finally, a correlation of firing between the multiple presynaptic interneurons that excite aCC/RP2 (Baines, 2003). In an attempt to distinguish between whether the increase in synaptic current ampli- 
Ai control

$\mathrm{Bi}$ postsynaptic
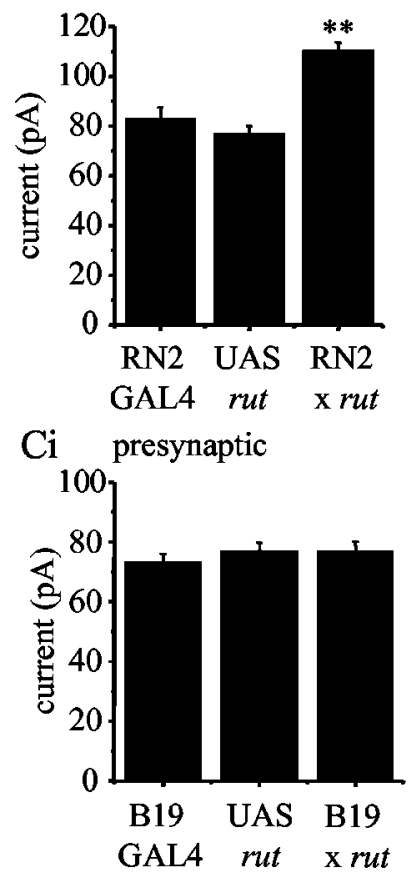

Di postsynaptic (+TTX)

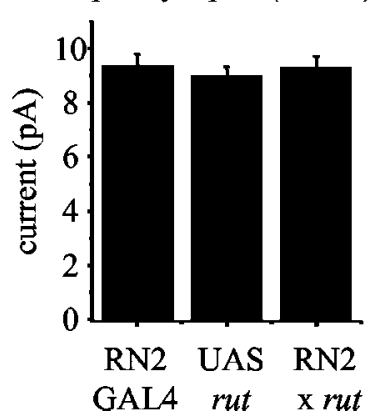

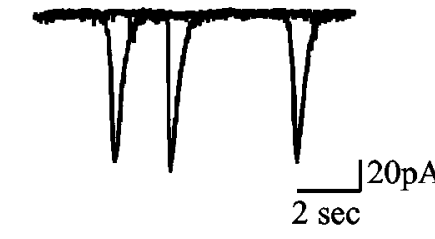

$\mathrm{Ci}$ presynaptic
Aii RN2 x UAS-rut

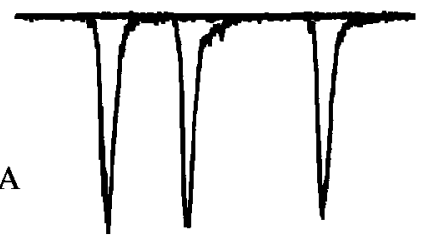

Bii

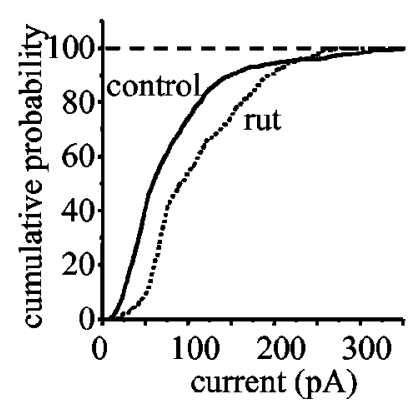

\section{Cii}

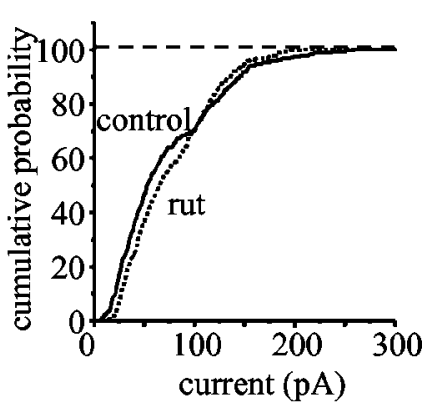

Dii

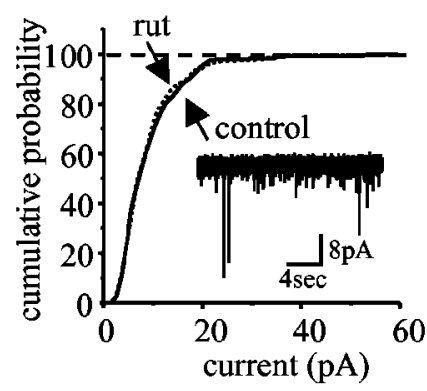

Figure 1. Postsynaptic cAMP increases synaptic excitation of aCC/RP2. Ai, Whole-cell voltage-clamp recordings $\left(V_{h},-60 \mathrm{mV}\right)$ from aCC/RP2 reveal large inward currents that are the result of the evoked release of presynaptic ACh (Baines et al., 1999, 2001). Aii, These currents are significantly increased in amplitude after overexpression of rut in aCC/RP2. Each panel shows three successive currents overlayed. Bi, The averaged amplitude of excitatory currents recorded in aCC/RP2 is significantly increased ( $p \leq 0.01$ ) after postsynaptic expression of UAS-rut compared with controls ( $C$, parental stocks). Bii, Cumulative probability plots of individual excitatory currents reveals a clear increase in amplitude after overexpression of rut in aCC/RP2. Ci, Cii, Targeted expression of rut to cholinergic neurons (B19-GAL4) does not effect the amplitude of synaptic currents recorded in aCC/RP2. Di, Dii, In the presence of TTX $(0.1 \mu \mathrm{M})$, evoked excitatory currents are abolished, leaving only those currents that are elicited from the spontaneous release of vesicles in the presynaptic interneurons (Dii, inset; calibration: 4 pA, $0.2 \mathrm{sec}$ ). Spontaneous currents in aCC/RP2 are unaffected in amplitude after overexpression of rut in these motoneurons. For all values, $n>8$; mean $\pm \mathrm{SE}$.

tude results from increased presynaptic release or from increased postsynaptic sensitivity to $\mathrm{ACh}$, these experiments were repeated in the presence of TTX $(0.1 \mu \mathrm{M})$. Under these conditions, all evoked transmitter release is blocked. Analysis of the remaining miniature synaptic currents (mEPSCs) in aCC/RP2 show no difference in amplitude $(8.7 \pm 0.25 \mathrm{pA}, 389 / 8$ vs $9.3 \pm 0.3 \mathrm{pA}, 265 / 8$ $\mathrm{pA} ; p>0.05$ ) after overexpression of rut in these neurons (Fig. $1 D)$. This is consistent with there being no change in postsynaptic sensitivity to released neurotransmitter. Frequency of mEPSCs was also not significantly different $(15 \pm 4.2$ vs $18 \pm 5$ per minute; control vs rut; $p>0.05$ ), suggestive that additional synaptic contacts have not formed between presynaptic interneurons and aCC/RP2. It can be concluded from these results that postsynaptic overexpression of UAS-rut is sufficient to either increase the probability of evoked presynaptic release of ACh or, alternatively, acts to better correlate the firing activity of convergent interneurons. Importantly, both mechanisms implicate the existence of retrograde signaling between postsynaptic motoneurons and presynaptic interneurons.

Overexpression of UAS-rut in aCC/RP2 is likely to activate PKA, through an increase in cAMP (Baines, 2003). To test whether this kinase is able to influence synaptic transmission, transgenes that either mimic or block PKA activity were expressed in these motoneurons. Postsynaptic expression of a constitutively active form of PKA $\left(P K A a c t^{l}\right)$, is sufficient to increase averaged synaptic current amplitude $(81.9 \pm 4.2 \mathrm{pA}, 385 / 8$ vs $98 \pm 2.4 \mathrm{pA}, 475 / 11 ; p \leq 0.01 ; \mathrm{RN} 2$ vs RN2 $\times \mathrm{UAS}^{-}$PKAact $\left.{ }^{1}\right)$ (Fig. 2). In comparison, inhibition of this kinase, by expression of PKAinh ${ }^{1}$, resulted in a significant reduction in averaged synaptic current amplitude $(81.9 \pm 4.2 \mathrm{pA}, 385 / 8$ vs $68 \pm 1.4 \mathrm{pA}, 410 / 9$; $p \leq 0.01$; RN2 vs RN2 $\times \mathrm{UAS} P K A$ inh $\left.^{l}\right)$ (Fig. 2$)$. These latter results lend additional support for the involvement of a retrograde signal but, more importantly, demonstrate that the mechanism that controls transmission at these identified synapses can both increase, as well as decrease, synaptic strength.

\section{Activation of BMP receptors in presynaptic interneurons is} sufficient to enhance synaptic excitation

Recent studies at the Drosophila NMJ implicate the BMP homolog Gbb and both type I and type II BMP receptors to be involved in retrograde signaling that is essential for proper synaptic growth and function (Aberle et al., 2002; Marqués et al., 2002; McCabe et al., 2003; Rawson et al., 2003). To test whether the retrograde signaling mechanism between central motoneurons and their presynaptic interneurons uses the same molecules, constitutively active type I receptors $\operatorname{sax}(\operatorname{sax} A)$ and $t k v(t k v A)$ were expressed either presynaptically or postsynaptically.

Of the two receptor constructs tested, only GAL4-mediated expression of activated $t k v$ in presynaptic cholinergic neurons (B19 GAL4) is sufficient to produce a large and significant increase in synaptic current amplitude in aCC/RP2 (Fig. 3A). This finding is complicated by the observation, however, that both the parental UAS-saxA and UAS- $t k v A$ lines exhibited a significant increase ( $p=0.02$ and 0.008 , respectively) in current amplitude compared with the GAL4 control. The reason for this is unknown but it is possible that these UAS transgenes may have a significant amount of basal expression in the absence of a GAL4 driver (Rawson et al., 2003). It is unlikely to be attributable to either genetic background or P-element insertion because both transgenes exhibit increased synaptic transmission. Significant levels of basal expression have been observed for other TGF- $\beta$ transgenes, including UAS- $g b b$ (McCabe et al., 2003, see below). Because of this, basal expression may occlude any specific GAL4-driven effect of activated saxA. GAL4-driven expression of activated $t k v A$, however, produces a clear and highly significant increase in synaptic current amplitude above that observed in UAS parental controls $(72.4 \pm 3.1 \mathrm{pA}, 272 / 8$ and $110 \pm 2.3 \mathrm{pA}, 417 / 8$ vs $166 \pm$ 
A

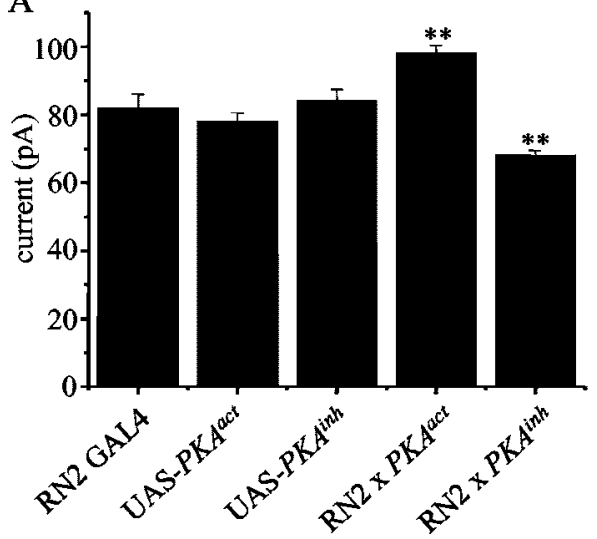

B

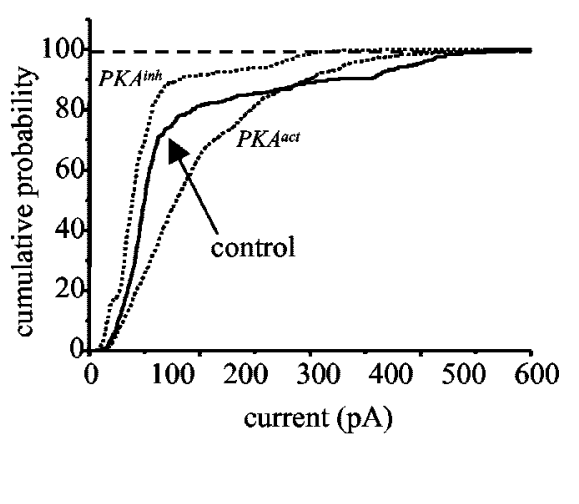

Figure 2. Postsynaptic PKA increases synaptic excitation of aCC/RP2. A, Postsynaptic expression (RN2-0 GAL4) of a constitutively active PKA transgene (PKA ${ }^{\text {act }}$ ) increases the amplitude of synaptic excitatory currents recorded in aCC/RP2 $(p \leq 0.01)$. In contrast, postsynaptic expression of a dominant-negative PKA (PKA $\left.{ }^{\text {inh }}\right)$ reduces the amplitude of these currents $(p \leq 0.01)$. Values are mean $\pm \mathrm{SE} ; n=8$. $B$, Cumulative probability plots of individual excitatory currents recorded after overexpression of $P K A^{\text {act }}$ and PKA ${ }^{\text {inh }}$ in aCC/RP2. Control is parental GAL4 and both UAS lines.

$6.5 \mathrm{pA}, 362 / 8 ; p<0.001 ; \mathrm{B} 19$ and UAS- $t k v A$ vs B19 $\times$ UAS- $t k v A)$ (Fig. $3 A, B$ ). Cumulative probability plots for individual currents in both control (B19 GAL4 and UAS-tkvA) and $t k v A$ expression $($ B19 GAL4 $\times$ UAS-tkvA) show a pronounced right shift for the latter (Fig. 3B). Such a shift mimics that seen for postsynaptic expression of rut. In the presence of TTX, mEPSC current amplitude was not affected by expression of $t k v A(7.0 \pm 0.23 \mathrm{pA}, 332 / 8$ vs $6.9 \pm 0.24 \mathrm{pA}, 295 / 8 \mathrm{pA} ; p>0.05$ ) (Fig. $3 B$, inset). Frequency of mEPSCs was also not significantly different $(13.7 \pm 4.4$ vs $14.1 \pm 2.3$ per minute; control vs experimental).

In contrast, GAL4-driven postsynaptic expression (RN2GAL4) of either activated $t k v$ or sax in aCC/RP2 had no effect on synaptic current amplitude in these motoneurons relative to parental control lines (Fig. 3C). Such a lack of effect is entirely consistent with a retrograde signaling mechanism in which a ligand is released postsynaptically to activate receptors localized presynaptically. These data implicate that $t k v$, a receptor for BMP homologs, may transduce the effect of such a retrograde signal.

\section{Overexpression of $g b b$ in postsynaptic motoneurons is sufficient to enhance synaptic excitation}

The observation that expression of an activated form of $t k v$ is sufficient to mimic the synaptic strengthening that results when rut is overexpressed in aCC/RP2 implicates that the retrograde signal is likely to be a BMP homolog. To test this possibility, two fly BMP homologs, $g b b$ and $d p p$, were expressed either presynaptically or postsynaptically. Postsynaptic expression of $g b b$, in aCC/RP2, results in highly significant synaptic strengthening between these motoneurons and their presynaptic interneurons (Fig. 4A). Again, as observed for the activated BMP receptors sax and $t k v$, the presence of UAS- $g b b$ alone is sufficient to produce a significant increase in averaged synaptic current amplitude. Indeed, the presence of this transgene alone (without a GAL4 driver) has previously been shown to be sufficient to rescue lethality in $g b b^{1} / g b b^{2}$ mutant larvae indicative of significant basal expression (McCabe et al., 2003). Quantification (real time RTPCR) of $g b b$ mRNA in WT and homozygous UAS- $g b b$ embryos confirms this by showing an approximately threefold increase in the latter (C. J. Mee, unpublished data). The increase in current amplitude seen in the presence of RN2-GAL4 is, however, considerably greater than that seen in UAS- $g b b$ alone $(81.9 \pm 4.2 \mathrm{pA}$,
$385 / 8$ and $103 \pm 3.8 \mathrm{pA}, 300 / 7$ vs $166 \pm$ $5.0 \mathrm{pA}, 304 / 8 ; p \leq 0.001$; RN2 and UAS$g b b$ vs RN2 $\times$ UAS- $g b b$ ) (Fig. $4 A$ ). Cumulative probability plots of individual currents are similar to those seen for presynaptic expression of $t k v A$, being indicative of most, if not all, presynaptic inputs to aCC/RP2 being potentiated. Unlike the effect of presynaptic expression of $t k v A$, however, the average amplitude of mEPSCs was decreased by expression of $g b b$ in aCC/RP2 $(8.7 \pm 0.25 \mathrm{pA}, 389 / 8$ vs $6.0 \pm 0.2 \mathrm{pA}, 164 / 8 ; p \leq 0.01$ ) (Fig. $4 B$, inset). Frequency of mEPSCs was not significantly different ( $15 \pm 4.2$ vs $11.7 \pm$ 2.1 per minute; control vs experimental). Because the increase in evoked synaptic current amplitude is considerable, the reduction in spontaneous currents may implicate the existence of additional postsynaptic compensatory mechanisms to prevent overexcitation of

motoneurons.

Expression of $g b b$ in presynaptic interneurons (B19 GAL4), by comparison, did not influence synaptic current amplitude (Fig. 4C). This, again, is a predictable outcome and serves to validate the hypothesis of retrograde signaling at this identified synapse. Thus, the predicted model consistent with these data implicates Gbb secretion to be required at appropriate sites of synaptic connectivity and not just bathing the neuropil in general, as might be predicted to result from a more generalized expression (i.e., B19-GAL4). This retrograde signaling mechanism also displays specificity of ligand because postsynaptic expression of UAS- $d p p$, another BMP ligand, failed to affect synaptic current amplitude (data not shown). Thus, taken together, these data support the hypothesis that increased adenylate cyclase activity in postsynaptic motoneurons is sufficient to strengthen synaptic transmission through the synapse-specific release of Gbb and, in turn, activation of its receptors $(t k v)$ in presynaptic cholinergic interneurons. Such a hypothesis is testable.

\section{Gbb is required for retrograde signaling}

Two significant predictions of the hypothesis that Gbb mediates a retrograde signal from motoneurons to interneurons are that first, endogenous synaptic strength and second, synaptic potentiation caused by increased postsynaptic adenylate cyclase activity, should be significantly diminished in the absence of this ligand. Analysis of synaptic current amplitude in the null alleles $g b b^{1}$ and $g b b^{2}$ show a clear and significant reduction in synaptic current amplitude compared with their heterozygous counterparts $\left(g b b^{1}: 54 \pm 0.9 \mathrm{pA}, 593 / 11\right.$ vs $79.5 \pm 2.2 \mathrm{pA}, 289 / 8 ; g b b^{2}$ : $53 \pm 2.3 \mathrm{pA}, 312 / 8$ vs $82 \pm 3.4 \mathrm{pA}, 228 / 8 ; p \leq 0.01$ for both alleles) (Fig. 5A). Thus, the first prediction is substantiated. To test the second prediction, UAS-rut was overexpressed in aCC/RP2 in a $g b b^{1}$ null background. Such expression fails to potentiate synaptic current amplitude $(54 \pm 0.9 \mathrm{pA}, 593 / 11$ vs $55 \pm 0.9 \mathrm{pA}, 611 / 12$; $p>0.05 ; g b b^{1}$ vs RN2 $\times$ UAS-rut) (Fig. $5 A, B$ ), thereby substantiating the second prediction. Thus, Gbb is both necessary and sufficient to facilitate synaptic strengthening, via retrograde signaling, between motoneurons and cholinergic presynaptic interneurons in the Drosophila CNS. 

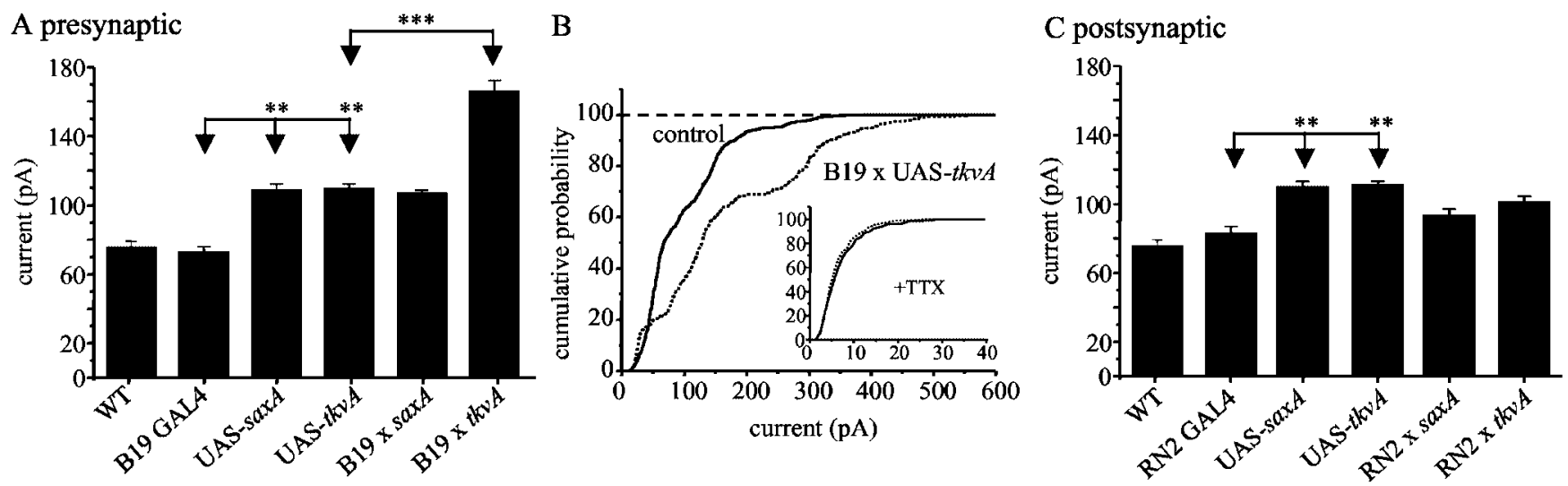

Figure 3. Presynaptic expression of activated $t k v$ is sufficient to potentiate synaptic transmission. $A$, Targeted expression (B19-GAL4) of activated tkv (tkvA) is sufficient to markedly increase the amplitude of evoked synaptic currents recorded in aCC/RP2 compared with parental controls $(p \leq 0.001)$. Parental UAS-tkvA stocks also exhibit significantly increased synaptic currents compared with B19-GAL4 ( $p \leq 0.01$ ). By comparison, GAL4-mediated expression of activated sax (saXA) shows no further increase in synaptic current amplitude above that seen in UAS-saxA controls (which again are significantly increased compared with B19-GAL4; $p \leq 0.01$ ). B, Cumulative probability plots show a marked shift to larger amplitudes for almost all currents recorded after GAL4-mediated expression of tkvA compared with parental controls (GAL4/UAS). A similar plot for spontaneous mEPSC amplitudes shows no such significant differences (inset). C, Postsynaptic expression (RN2-GAL4) of either tkvA or saxA does not increase synaptic current amplitude in aCC/RP2, above that seen in UAS controls. For all values, $n>8$; mean \pm SE.

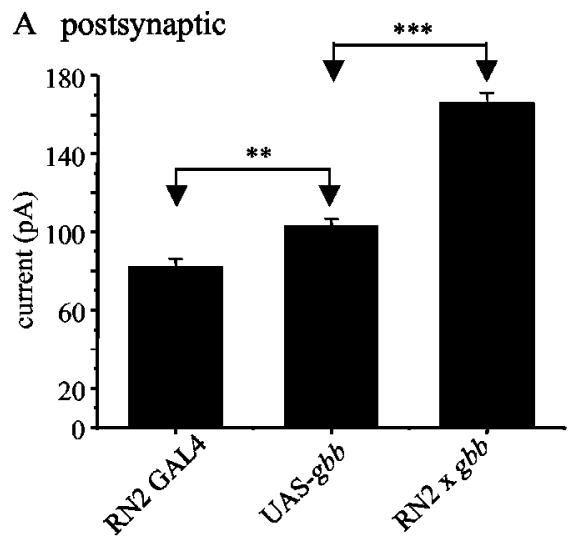

B

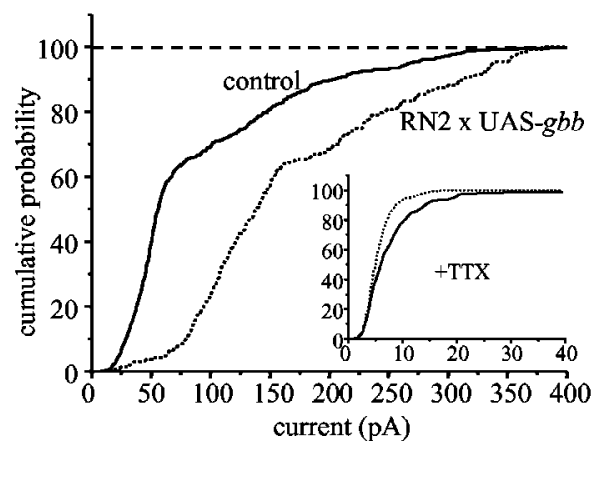

C presynaptic

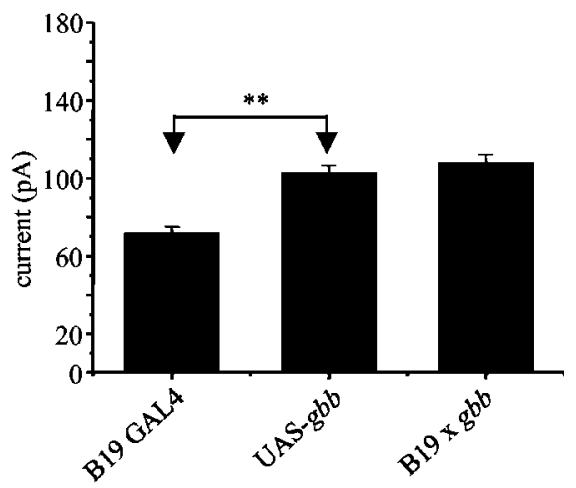

Figure 4. Postsynaptic expression of $g b b$ is sufficient to increase the amplitude of synaptic currents. $A$, Targeted expression (RN2-GAL4) of $g b b$ in aCC/RP2 is sufficient to greatly increase the amplitude of evoked synaptic currents recorded ( $p \leq 0.001$ ), compared with the UAS control, which is also significantly increased compared with RN2-GAL4 ( $p \leq 0.01)$. B, Cumulative probability plots show a large shift to the right for evoked currents observed in aCC/RP2 that are overexpressing gbb compared with control (GAL4/UAS). In the presence of TTX, spontaneous mEPSC amplitudes are, in contrast, significantly smaller because of overexpression of $g b b$ in aCC/RP2 (inset). C, Targeted expression of $g b b$ to cholinergic neurons (B19-GAL4) fails to increase synaptic current amplitude in aCC/RP2, above that already observed for UAS-gbb alone. For all values, $n>8$; mean \pm SE.

Potentiation of synaptic transmission by $\mathrm{Gbb}$ is activity dependent

Potentiation of synaptic transmission, produced by postsynaptic overexpression of $g b b$, may or may not be activity dependent. In the former, only active synapses would be potentiated, whereas in the latter, constitutive secretion of Gbb might consolidate all synaptic connections regardless of their activity state. To differentiate between these two possibilities, $g b b$ was overexpressed, in aCC/RP2, in a background that allowed cholinergic synaptic transmission to be manipulated. The temperature-sensitive allele of choline acetyltransferase (Chat), ch $a^{t s 2}$, allows cholinergic transmission to be blocked by shifting to a restrictive temperature of $29^{\circ} \mathrm{C}$ (Baines et al., 2001). Even at the permissive temperature $\left(20^{\circ} \mathrm{C}\right.$ and below), the activity of Chat in this allele is significantly less than WT (Salvaterra and McCaman, 1985). Averaged synaptic current amplitude, recorded in aCC/RP2, in $c h a^{t s 2}$ larvae (raised at $20^{\circ} \mathrm{C}$ ) is significantly reduced compared with WT consistent with the notion that potentiation of transmission at these synapses is, at least, partially dependent on normal levels of cholinergic synaptic activity (Fig. 6A). This is because even at the permissive temperature, Chat activity (and hence cholinergic transmission) is reduced relative to WT animals (Salvaterra and McCaman, 1985). This assumption is strongly supported by the parallel finding that postsynaptic expression of $g b b$, in aCC/RP2, in a $c h a^{t s 2}$ background fails to elicit synaptic potentiation when raised at $29^{\circ} \mathrm{C}$ (restrictive temperature) compared with heterozygous controls exposed to identical developmental conditions (cha $a^{t s 2} /$ FM7) (Fig. 6A). The potentiation of current amplitude caused by postsynaptic expression of $g b b$ in heterozygous controls is, however, again smaller than that seen for similar genetic manipulations in a WT background (compare Fig. 4A).

To further demonstrate a requirement for activity in Gbbdependent synaptic potentiation, embryos in which $g b b$ was overexpressed in aCC/RP2 in $c h a^{t s 2}$ and developed at $29^{\circ} \mathrm{C}$ (sufficient to prevent potentiation, see above), were then shifted back to $20^{\circ} \mathrm{C}$ and allowed to develop for an additional $\sim 22 \mathrm{hr}$. Analysis of synaptic current amplitude in these late first instar larvae was significantly increased compared with $c h a^{t s 2}$ counterparts exposed to the same developmental paradigm (Fig. $6 B$ ). Thus, suppression of cholinergic signaling is sufficient to prevent synaptic 


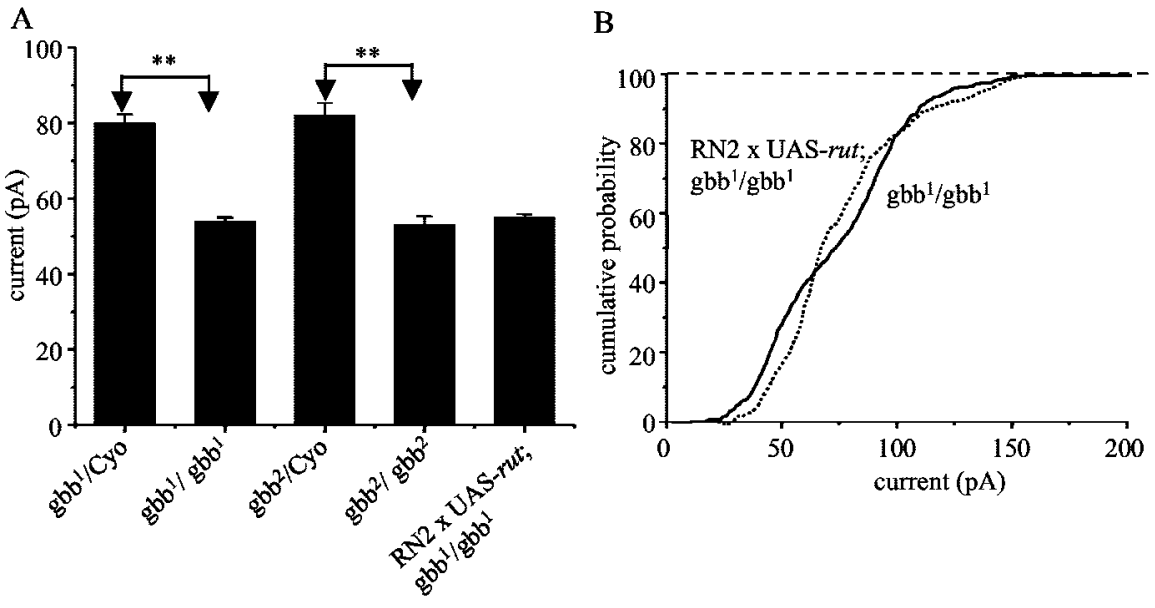

Figure 5. Gbb is necessary for synaptic strengthening. A, Evoked synaptic current amplitude recorded in aCC/RP2 is significantly smaller in null alleles of gbb ( $g b b^{1}$ and $g b b^{2}$ ) compared with heterozygous controls (allele/Cyo::GFP balancer; $p \leq 0.01$ ). Moreover, postsynaptic expression of rut, which is normally sufficient to significantly increase evoked synaptic currents in aCC/RP2 (Fig. $1 A, B)$, fails to do so in the absence of $g b b . B$, Cumulative plots of evoked synaptic current amplitude reinforce the observation that in the absence of $g b b$, targeted expression (RN2-GAL4) of rut in aCC/RP2 fails to increase current amplitude above that seen in either null allele of $g b b$ examined ( $g b b^{7}$ shown).
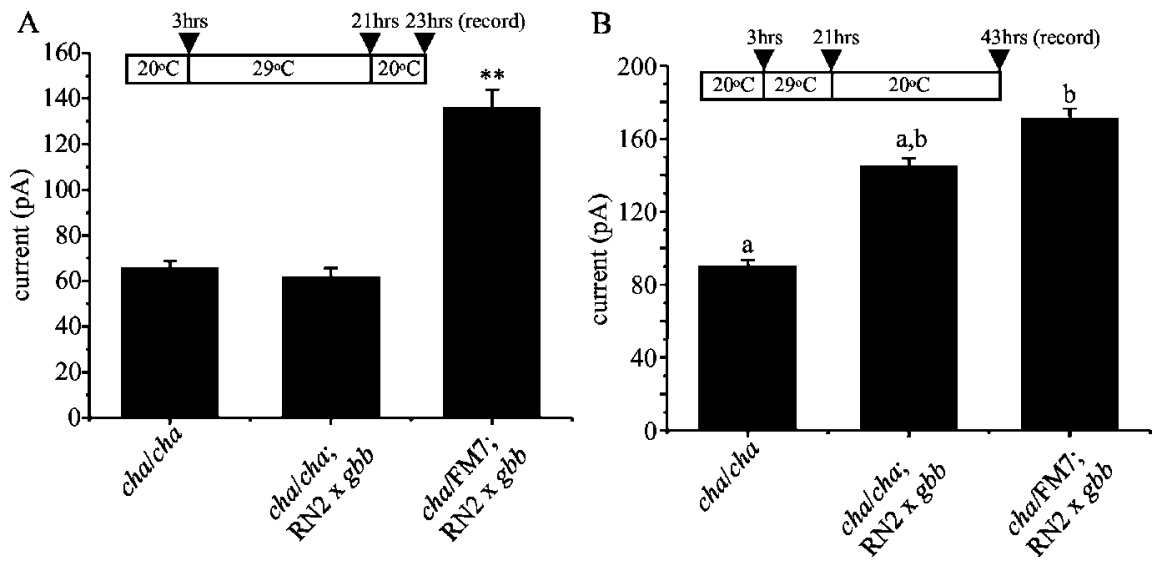

Figure 6. Gbb signaling is activity dependent. $A$, After a $3 \mathrm{hr}$ period of egg laying at $20^{\circ} \mathrm{C}$, cha ${ }^{t s 2}$ embryos were shifted to $29^{\circ} \mathrm{C}$ and allowed to develop to late stage 17 (Baines et al., 2001). Before recording synaptic currents in aCC/RP2, embryos were transferred back to $20^{\circ} \mathrm{C}$ for an additional $2 \mathrm{hr}$, during which time hatching occurred. Recordings of current amplitude under these conditions reveal that postsynaptic expression of $g b b$ (using RN2-0 GAL4) is unable to potentiate synaptic current amplitude in the absence of cholinergic signaling. By comparison, heterozygous controls (chats2/FM7), in which cholinergic signaling is functional, which also carried both RN2-0 GAL4 and UAS-gbb, showed significantly larger synaptic currents compared with $c h a^{t s 2}(p \leq 0.01)$. $B$, Restoration of cholinergic function for extended periods $(\sim 22 \mathrm{hr})$ in these same larvae is sufficient to restore the ability of postsynaptic expression of $g b b$ to potentiate synaptic current amplitude (identical letters indicate $p \leq 0.01$ ). For all values, $n>6$; mean $\pm S E$

strengthening caused by postsynaptic expression of $g b b$, an effect that can be reversed by restoration of cholinergic function. Taken together, these data clearly demonstrate a requirement for neuronal activity for Gbb-dependent potentiation of synaptic transmission. Whether activity is required for secretion of postsynaptic Gbb or the proper signaling of presynaptic activated receptors cannot be distinguished.

\section{Discussion}

Previous studies implicate BMP signaling to be essential for the correct growth and function of the NMJ, in addition to the expression of circulating neurohormones that are, themselves, capable of modulating the physiology of the NMJ (Aberle et al., 2002; Marqués et al., 2002, 2003; Allan et al., 2003; McCabe et al., 2003; Rawson et al., 2003). The findings of the present study extend further the role of BMP signaling to include sites of central synaptic connectivity within the circuits that control motoneuron function. Thus, taken together, BMP signaling, principally mediated by Gbb, seemingly acts to integrate both the development and physiology of the neuronal circuitry that controls motor behavior in Drosophila.

\section{BMP signaling in Drosophila}

BMP signaling has been well characterized and, moreover, is highly conserved across diverse species (Massague and Wotton, 2000). Drosophila has at least two BMP type I receptors, sax and $t k v$, and two type II receptors, wit and punt. There are also two R-Smads, mothers against decapentaplegic (mad) and dSmad2. The greatest diversity is, however, exhibited in BMP ligands with seven known: namely, gbb, $d p p$, screw, activin, activin-like protein, myoglianin, and maverick (Lo and Frasch, 1999; Nguyen et al., 2000; Keshishian and Kim 2004). The ligand Gbb, along with type I receptors Sax and Tkv and the type II receptor Wit, have been shown to be part of a retrograde signaling mechanism that ensures correct growth and function of the NMJ (Aberle et al., 2002; Marqués et al., 2002; McCabe et al., 2003; Rawson et al., 2003). In the absence of either $g b b$ or wit, NMJs exhibit small synapses with aberrant ultrastructure, in addition to reduced levels of evoked neurotransmitter release. Rescue of this phenotype is complex. Restoration of $g b b$ expression in muscles is sufficient to rescue synaptic structure but is only weakly able to rescue synaptic function. Full rescue of synaptic function is only achieved by pan-neuronal expression of UAS- $g b b$ (McCabe et al., 2003), indicative of an additional role for Gbb signaling within the CNS.

The expression of FMRF-amide (FMRFa)-like peptides in a subset of central peptidergic neurons, termed Tv neurons, is similarly dependent on Gbb retrograde signaling from neurohaemal organs innervated by these neurons (Allan et al., 2003; Marqués et al., 2003). Through a particularly elegant use of chimeric receptors, Marqués et al. (2003) provide convincing data to indicate that Gbb acts via Wit and Tkv to specify FMRFa expression. Chimeric receptors containing elements from both Wit and Sax were nonfunctional. Similar results have recently been obtained at the NMJ by McCabe et al. (2004) that serve to underline the fact that although Sax is seemingly required for correct NMJ development, it is not sufficient to transmit the Wit signal. In the absence of sax, NMJs exhibit reductions in both synaptic size and neurotransmission, a phenotype that is, somewhat perplexingly, recapitulated by pan-neuronal expression of DN $t k v$ but not by DNsax (Rawson et al., 2003; McCabe et al., 2004). Thus, although there are details that remain to be clarified, clearly BMP signaling at the 
NMJ is essential for normal synaptic development and, moreover, is reliant on retrograde signaling that involves $g b b, s a x, t k v$, and wit. Within the CNS, the results of this present study implicate that postsynaptic Gbb signaling might be transduced through $t k v$. The observation that activated sax is unable to potentiate synaptic transmission above that seen in the UAStransgenic parent cannot, however, be taken as definitive evidence that this type I BMP receptor is not involved in synaptic development at this central synapse. Additional studies using loss of function mutants will be required to assess the contribution of this, and other BMP receptors, within the CNS.

The mechanism that underlies BMP signaling in the CNS remains unknown. At the NMJ, BMP signaling is seemingly mediated through Wit-dependent phosphorylation of R-Smads in the presynaptic motoneurons (Marqués et al., 2002; McCabe et al., 2003, 2004; Rawson et al., 2003). These studies failed, however, to show P-Mad reactivity in central interneurons which, in the light of the present findings, might be indicative of differing transduction mechanisms centrally compared with peripherally. A similar conclusion could be drawn from the observation that synaptic excitation of aCC/RP2 is normal in loss of function alleles of mad ( $\mathrm{mad}^{1}$ and $\mathrm{mad}^{12}$ ) (my unpublished data); these same alleles show reduced synaptic transmission at the NMJ (Rawson et al., 2003). Alternative, non-Mad, signaling pathways that transduce BMP signals are documented (e.g., activation of Ras, RhoB, TAK1 and protein phosphatase 2A) (Derynck et al., 2001) but remain to be characterized in detail. Caution should prevail, however, before any conclusions about BMP signal transduction within the CNS are drawn. Considerable additional studies will be required, including the targeted expression of Mad transgenes, before the involvement of R-Smads in motoneuron to interneuron BMP-dependent retrograde signaling can be evaluated. The role of R-Smads at the NMJ is also far from established, particularly because P-mad accumulation in motoneurons is strong in early embryos but undetectable in late third instar larvae when electrophysiology shows that BMP signaling is active (Marqués et al., 2002).

\section{Gbb signaling is activity dependent}

An important issue, which has not been addressed in previous studies, is how Gbb signaling is regulated. There are two probable models. In the first, Gbb-mediated signaling, and/or secretion, is primarily constitutive. Which synapses are affected by such constitutive signaling would thus likely be a property intrinsic to the presynaptic element. The second model, however, requires that signaling be linked to presynaptic activity. A third possibility would allow both models to operate, with activity of the presynapse acting to increase retrograde signaling above that of the constitutive level. The trophic hypothesis has been proposed to account for activity-dependent synaptic remodeling observed in developing vertebrate nervous systems, particularly in complex sensory circuitry such as the visual and auditory cortex (for review, see Fitzsimonds and Poo, 1998). Retrograde signaling by implicated neurotrophins can be coupled to electrical activity in a variety of ways. Expression, secretion, receptor binding, and/or signal transduction could each or all be activity dependent. There is considerable evidence to show that expression of individual neurotrophins is activity dependent (for review, see Lindholm et al., 1994; Fitzsimonds and Poo, 1998). Secretion of the neurotrophin, nerve growth factor (NGF), is known to be both constitutive and regulated in rat hippocampus. The regulated pathway can be triggered by exposure to high $\mathrm{K}^{+}$, carbachol, or glutamate, indicative that it is activity dependent. Moreover, regulated se- cretion is dependent on extracellular $\mathrm{Na}^{+}$, but surprisingly not $\mathrm{Ca}^{2+}$, and is blocked by TTX (Blochl and Thonen, 1995, 1996). Although the release of NGF and other neurotrophins is clearly activity dependent, uptake of the released signal may also depend on the level of activity in the presynapse. Presynaptic terminals undergoing higher levels of synaptic release are believed to be more likely to internalize neurotrophin receptor complexes than adjacent quiescent terminals (Fitzsimonds and Poo, 1998). Such discrimination would provide a degree of specificity in models that envisage constitutive secretion.

Two observations implicate that retrograde signaling mediated by Gbb is, at least in part, activity dependent in Drosophila CNS. The first is that Gbb signaling lies downstream of increased adenylate cyclase activity, itself likely to result in increased cAMP, a second messenger known to be increased after postsynaptic depolarization (Yu et al., 2004). The second, and perhaps more convincing, is that when cholinergic transmission is prevented during embryogenesis, postsynaptic overexpression of $g b b$ fails to lead to a potentiation of synaptic transmission, an effect that is reversible on restoration of normal synaptic function. Measurement of $g b b$ expression using quantitative real time RT-PCR fails to reveal any dependence on activity (C. Mee and R. A. Baines, unpublished data). Thus, it would seem more likely that postsynaptic activity is able to increase either Gbb secretion and/or transduction of the signal once receptor activation has occurred.

In summary, many levels of the motor system of Drosophila are seemingly reliant on retrograde signaling mediated by Gbb. In the periphery, such signaling is required for appropriate development and function of the NMJ. Centrally, expression of FMRFalike peptides that, in turn, modulate NMJ physiology also requires Gbb retrograde signaling. This present study demonstrates that the central synapses that coordinate motoneuron activation are also reliant on Gbb-dependent retrograde signaling. It is conceivable that conservation of retrograde ligand facilitates the integration of entire neuronal networks, regardless of the precise site of release. For example, overexpression of $g b b$ in body wall muscle is seemingly sufficient to strengthen central synapses between interneurons and aCC/RP2 (my unpublished data). However, whether this is attributable to translocation of retrograde signal through motoneurons remains to be determined. Of particular interest now will be the determination of whether neuronal circuits distinct from the motor system use Gbb or one of the other BMP ligands present in the Drosophila genome, if at all.

\section{References}

Aberle H, Haghighi AP, Fetter RD, McCabe BD, Magalhães TR, Goodman CS (2002) Wishful thinking encodes a BMP type II receptor that regulates synaptic growth in Drosophila. Neuron 33:545-558.

Allan DW, St Pierre SE, Miguel-Aliaga I, Thor S (2003) Specification of neuropeptide cell identity by the integration of retrograde BMP signalling and a combinatorial transcription factor code. Cell 113:73-86.

Baines RA (2003) Postsynaptic protein kinase A reduces neuronal excitability in response to increased synaptic excitation in the Drosophila CNS. J Neurosci 23:8664-8672.

Baines RA, Bate M (1998) Electrophysiological development of central neurons in the Drosophila embryo. J Neurosci 18:4673-4683.

Baines RA, Robinson SG, Fujioka M, Jaynes JB, Bate M (1999) Postsynaptic vesicle release is essential for synaptogenesis in Drosophila. Curr Biol 9:1267-1270.

Baines RA, Uhler JP, Thompson A, Sweeney ST, Bate M (2001) Altered electrical properties in Drosophila neurons developing without synaptic transmission. J Neurosci 21:1523-1531.

Blochl A, Thonen H (1995) Characterization of nerve growth factor (NGF) release from hippocampal neurons: evidence for a constitutive and an unconventional sodium-dependent regulated pathway. Eur J Neurosci $7: 1220-1228$ 
Blochl A, Thonen H (1996) Localization of cellular storage compartments and sites of constitutive and activity-dependent release of nerve growth factor (NGF) in primary cultures of hippocampal neurons. Mol Cell Neurosci 7:173-190.

Davis GW, DiAntonio A, Petersen SA, Goodman CS (1998) Postsynaptic PKA controls quantal size and reveals a retrograde signal that regulates presynaptic transmitter release in Drosophila. Neuron 20:305-315.

Derynck R, Akhurst RJ, Balmain A (2001) TGF- $\beta$ signalling in tumor suppression and cancer progression. Nat Genet 29:117-129.

Fitzsimonds RM, Poo M-M (1998) Retrograde signalling in the development and modification of synapses. Physiol Rev 78:143-170.

Fujioka M, Emi-Sarker Y, Yusibova GL, Goto T, Jaynes JB (1999) Analysis of an even-skipped rescue transgene reveals both composite and discrete neuronal and early blastoderm enhancers, and multi-stripe positioning by gap gene repressor gradients. Development 126:2527-2538.

Keshishian H, Kim Y-S (2004) Orchestrating development and function: retrograde BMP signalling in the Drosophila nervous system. Trends Neurosci 27:143-147.

Lindholm D, Castren E, Berzaghi M, Blochl A, Thonen H (1994) Activitydependent and hormonal regulation of neurotrophin mRNA levels in the brain: implications for neuronal plasticity. J Neurobiol 25:1362-1372.

Lo PC, Frasch M (1999) Sequence and expression of myoglianin, a novel Drosophila gene of the TGF-beta superfamily. Mech Dev 86:171-175.

Marqués G, Bao H, Haerry TE, Shimell MJ, Duchek P, Zhang B, O’Connor MB (2002) The Drosophila BMP type II receptor wishful thinking regulates neuromuscular synapse morphology and function. Neuron 33:529-543.

Marqués G, Haerry TE, Crotty ML, Xue M, Zhang B, O’Connor MB (2003) Retrograde Gbb signalling through BMP type 2 receptor wishful thinking regulates systemic FMRFa expression in Drosophila. Development 130:5457-5470.

Massague J (1998) TGF-beta signal transduction. Annu Rev Biochem 67:753-791.

Massague J, Wotton D (2000) Transcriptional control by the TGF- $\beta / \mathrm{Smad}$ signalling system. EMBO J 19:1745-1754.

McCabe BD, Marqués G, Haghighi AP, Fetter RD, Crotty ML, Haerry TE, Goodman CS, O'Connor MB (2003) The BMP homologue Gbb pro- vides a retrograde signal that regulates synaptic growth at the Drosophila neuromuscular junction. Neuron 39:241-254.

McCabe BD, Horn S, Aberle H, Fetter RD, Marqués G, Haerry TE, Wan H O'Connor MB, Goodman CS (2004) Highwire regulates presynaptic BMP signalling essential for synaptic growth. Neuron 41:891-905.

Nguyen M, Parker L, Arora K (2000) Identification of maverick, a novel member of the TGF-beta superfamily in Drosophila. Mech Dev 95:201-206.

Packard M, Koo ES, Gorczyca M, Sharpe J, Cumberledge S, Budnik V (2002) The Drosophila Wnt, wingless, provides an essential signal for pre- and postsynaptic differentiation. Cell 111:319-330.

Packard M, Matthew D, Budnik V (2003) Wnts and TGF $\beta$ in synaptogenesis: Old friends signalling at new places. Nat Rev Neurosci 4:113-120.

Rawson JM, Lee M, Kennedy EL, Selleck SB (2003) Drosophila neuromuscular synapse assembly and function require the TGF- $\beta$ type I receptor saxophone and the transcription factor mad. J Neurobiol 55:134-150.

Salvaterra PM, Kitamoto T (2001) Drosophila cholinergic neurons and processes visualized with Gal4/UAS-GFP. Gene Expr Patterns 1:73-82.

Salvaterra PM, McCaman RE (1985) Choline acetyltransferase and acetylcholine levels in Drosophila melanogaster: a study using two temperaturesensitive mutants. J Neurosci 5:903-910.

Sheng M, Kim MJ (2002) Postsynaptic signalling and plasticity mechanisms. Science 298:776-780.

Soderstrom S, Bengtsson H, Ebendal T (1996) Expression of serine/threonine kinase receptors including bone morphogenetic factor type II receptor in developing and adult rat brain. Cell Tissue Res 286:269-279.

Wharton KA, Cook JM, Torres-Schumann S, de Castro K, Borod E, Phillips DA (1999) Genetic analysis of the bone morphogenetic protein-related gene, gbb, identifies multiple requirements during Drosophila development. Genetics 152:629-640.

Yu X, Byrne JH, Baxter DA (2004) Modeling interactions between electrical activity and second messenger cascades in Aplysia neuron B15. J Neurophysiol 91:2297-2311.

Zars T, Wolf R, Davis R, Heisenberg M (2000) Tissue-specific expression of a type I adenylyl cyclase rescues the rutabaga mutant memory defect: in search of the engram. Learn Mem 7:18-31. 